\title{
Diacylglycerol kinase synthesized by commensal Lactobacillus reuteri diminishes protein kinase C phosphorylation and histamine-mediated signaling in the mammalian intestinal epithelium
}

BP Ganesh ${ }^{1,2,3}$, A Hall ${ }^{1,2}$, S Ayyaswamy ${ }^{1,2}$, JW Nelson ${ }^{4}$, R Fultz ${ }^{2,4}$, A Major ${ }^{1,2}$, A Haag $^{2}$, M Esparza $^{1,2}$, M Lugo ${ }^{1,2}$, S Venable ${ }^{1,2}, M_{\text {Whary }}^{3}$, JG Fox ${ }^{3}$ and J Versalovic ${ }^{1,2}$

Lactobacillus reuteri 6475 (Lr) of the human microbiome synthesizes histamine and can suppress inflammation via type 2 histamine receptor (H2R) activation in the mammalian intestine. Gut microbes such as Lr promote H2R signaling and may suppress H1R proinflammatory signaling pathways in parallel by unknown mechanisms. In this study, we identified a soluble bacterial enzyme known as diacylglycerol kinase (Dgk) from Lr that is secreted into the extracellular milieu and presumably into the intestinal lumen. DgK diminishes diacylglycerol (DAG) quantities in mammalian cells by promoting its metabolic conversion and causing reduced protein kinase $C$ phosphorylation ( $p P K C$ ) as a net effect in mammalian cells. We demonstrated that histamine synthesized by gut microbes (Lr) activates both mammalian H1R and H2R, but Lr-derived Dgk suppresses the H1R signaling pathway. Phospho-PKC and $\mathrm{I} B \alpha$ were diminished within the intestinal epithelium of mice and humans treated by wild-type (WT) Lr, but pPKC and IкB $\alpha$ were not decreased in treatment with $\triangle d g k A$ Lr. Mucosal IL-6 and systemic interleukin (IL)-1 $\alpha$, eotaxin, and granulocyte colony-stimulating factor (G-CSF) were suppressed in WT Lr, but not in $\triangle d g k A$ Lr colonized mice. Collectively, the commensal microbe Lr may act as a "microbial antihistamine" by suppressing intestinal H1R-mediated proinflammatory responses via diminished pPKC-mediated mammalian cell signaling.

\section{INTRODUCTION}

Diacylglycerol kinases (DGKs) are key enzymes in mammalian lipid metabolism that phosphorylate diacylglycerol (DAG) formed by the turnover of membrane phospholipids to phosphatidic acid (PA). ${ }^{1,2}$ Many mammalian hormones, growth factors, and other cell stimuli evoke a transient increase in the amounts of cellular DAG through hydrolysis of phosphoinositides by phospholipase $C^{3}$ upon $G$ proteincoupled receptor (GPCR) activation. ${ }^{4}$ As an example, when histamine binds to a $G$ protein-coupled histamine receptor, ${ }^{5}$ DAG is an established second messenger and an allosteric activator of protein kinase $\mathrm{C}(\mathrm{PKC})^{4,6,7}$ for important biological processes. ${ }^{3,8}$ In mammals, DAG is a precursor in phospholipid metabolism, but it also serves as an intracellular lipid signal that activates PKC and is involved in cell cycle regulation, cell survival, tumorigenesis, and apoptosis. ${ }^{1,9}$ DGK consumes DAG to produce $\mathrm{PA}$ and is a potential terminator of DAG signaling. By attenuating DAG levels, DGK may downregulate membrane localization of $\mathrm{PKC}$ and may terminate transient receptorinduced $\mathrm{PKC}$ activation, thereby inhibiting signaling pathways downstream of PKC. ${ }^{3}$

In bacteria, the head group of phosphatidylglycerol is extensively used in the biosynthesis of membrane components, and removal of the head group generates $\mathrm{DAG}^{8}{ }^{8} \mathrm{DAG}$ is converted to PA by $\mathrm{Dgk}^{2}$ and recycles DAG into the cytidine diphosphate-diacylglycerol pathway for phospholipid

${ }^{1}$ Departments of Pathology and Immunology, Baylor College of Medicine, Houston, Texas, USA. ²Department of Pathology, Texas Children's Hospital, Houston, Texas, USA. ${ }^{3}$ Division of Comparative Medicine, Department of Biological Engineering, Massachusetts Institute of Technology, Cambridge, Massachusetts, USA and ${ }^{4}$ Graduate Program in Integrative Molecular and Biomedical Sciences, Baylor College of Medicine, Houston, Texas, USA. Correspondence: BP Ganesh (bhanu.ganesh@bcm.edu) 
synthesis, thereby preventing the lethal accumulation of DAG in bacterial membranes. ${ }^{8}$ Lactobacillus reuteri 6475 (Lr) genome has been sequenced, ${ }^{10}$ and this bacterial genome contains a gene encoding diacylglycerol kinase (Dgk). Grampositive bacteria have been documented to express soluble isoforms of Dgk. ${ }^{8,11}$ As bacterial Dgk shares $19 \%$ sequence identity with mammalian DGK, ${ }^{8}$ we speculated that Lr-derived Dgk enzyme might inhibit DAG downstream signaling in bacterial and mammalian cells. ${ }^{3,8}$

A key biogenic amine, histamine, signals via type 1 histamine receptor (H1R) to affect PKC signaling in mammalian cells. Histamine serves a primary role as a mediator of allergic inflammation. ${ }^{12,13}$ Histamine is also a neurotransmitter that plays a key role in areas of the central nervous system enriched with histaminergic neurons such as the hippocampus. ${ }^{5,14,15}$ Apart from mammals, microbes can synthesize histamine to maintain intracellular $\mathrm{pH}^{16,17}$ One such bacterium, Lactobacillus reuteri 6475 , belongs to the phylum Firmicutes and is indigenous to the gastrointestinal tracts of avian and mammalian species. ${ }^{18}$ This organism is considered to be a GRAS (generally recognized as safe) and beneficial microbe, and has been used globally as a probiotic for more than two decades. A recent pangenomic study showed that $\operatorname{Lr} 6475$ strain is distinctive among gut microbes by containing a complete chromosomal $h d c$ gene cluster (genes $h d c A, h d c B$, and $h d c P$ ) and the genetic capacity to convert histidine to histamine. ${ }^{19}$ Lr-derived histamine suppressed proinflammatory cytokines in intestinal epithelial cells, monocytes, ${ }^{17,20}$ and intestinal inflammation in different rodent models. ${ }^{21,22}$ However, the molecular mechanisms behind the interactions of Lr-derived histamine and the mammalian intestinal epithelium are not clearly understood.

Histamine interacts with the host using four different mammalian receptors (H1R, H2R, H3R, and H4R). ${ }^{23}$ The majority of histamine receptors in the gut are known to be H1R and $\mathrm{H}_{2} \mathrm{R}^{24,25} \mathrm{H} 1 \mathrm{R}$ is known to initiate proinflammatory pathways by increasing phosphorylation of protein kinase C (pPKC) ${ }^{5,26-28} \mathrm{H} 2 \mathrm{R}$, in contrast, suppresses inflammation by inducing protein kinase A phosphorylation ${ }^{5,29}$ or by suppressing Toll-like receptor-mediated inflammation. ${ }^{30} \mathrm{H} 2 \mathrm{R}$ antagonists increased infection risk and necrotizing enterocolitis in low birth weight infants ${ }^{31,32}$ and doubled the risk of hospitalization and surgery for patients with Crohn's disease. ${ }^{33}$ Suppression of intestinal inflammation depended on the presence of dietary L-histidine and histamine-generating Lr 6475 in a murine colitis model via a H2R-mediated mechanism. ${ }^{34}$ Histidine decarboxylase (HDC) is the unique enzyme responsible for generation of a biogenic amine, histamine, via conversion of L-histidine in mammals, including humans. ${ }^{14,35}$ HDC deficiency in mice was shown to promote inflammation-associated colorectal cancer by accumulation of $\mathrm{CD} 11 \mathrm{~b}^{+} \mathrm{Gr}-1^{+}$immature myeloid cells (IMCs) in bone marrow and spleen compared with wild-type (WT) mice. ${ }^{14}$ This deficiency was associated with increased concentrations of proinflammatory cytokines, especially interleukin (IL)-6 and IL-1, and excessive quantities of IL-1 were generated at least partly by immature myeloid cells in the circulation. ${ }^{14}$ Histamine plays a key role in the maturation of IMCs via HDC expression. ${ }^{36}$

Histamine derived from Lr differentially promotes $\mathrm{H} 2 \mathrm{R}$ pathway signaling and may antagonize H1R signaling in parallel. Microbiome-generated Dgk synthesized by Lr may interact with host epithelial DAG and inhibit H1R downstream signaling by converting DAG to PA, thereby reducing quantities of DAG, preventing PKC phosphorylation, and likely reducing intestinal inflammation. To better understand the molecular interactions of a single commensal bacterium Lr with the intestinal epithelium and its impact on shaping gut immune maturation, the mammalian intestine was colonized with WT or mutant ( $\Delta h d c A$ or $\Delta d g k A$ ) Lr strains deficient in diacylglycerol kinase or histamine production using inbred and outbred germ-free (GF) mouse models. The results described in this study show that bacterial $\operatorname{DgK}$ is secreted into the extracellular milieu and modulates PKC signaling in mouse and human intestinal epithelial cells. Furthermore, microbial DAG kinase appears to modulate myeloid cell maturation and cytokine production in vivo.

\section{RESULTS}

\section{WT and mutant $L$. reuteri 6475 stably colonize the GF mouse} intestine

The 10-week-old BALB/c GF mice were either colonized with L. reuteri $6475 \mathrm{WT}(\mathrm{WT})$ or $\Delta h d c A$ L. reuteri $6475(\Delta h d c A)$ or $\triangle d g k A$ L. reuteri $6475(\triangle d g k A)$ on day 1 and maintained for 22 days (Figure 1a). On day 23, the mice were killed and intestinal contents were collected and processed as described. Extracted DNA was quantified using quantitative PCR. GF mice colonized for 22 days with WT or $\Delta h d c A$ or $\Delta d g k A$ Lr were successfully colonized with a single $\mathrm{Lr}$ administration. We detected $\log _{10} 8.56 \pm 0.72$ of WT Lr, $\log _{10} 7.64 \pm 0.76$ of $\Delta h d c A$, and $\log _{10} 7.06 \pm 0.66$ of $\Delta d g k A$ per gram wet weight of cecum contents of BALB/c gnotobiotic mice (Figure 1b). Similarly, 13-week-old Swiss Webster (SW) GF mice were stably colonized with L. reuteri 6475 WT (WT) for 22 days (Supplementary Figure S1a online). However, SW GF mice yielded more abundant gut lactobacilli following colonization by $\mathrm{Lr}$ compared with BALB/c GF mice. On day 23, the SW mice were killed, and were colonized with $\log _{10} 9.83 \pm 0.24$ of WT Lr per gram of cecal contents (Supplementary Figure S1b).

\section{Systemic immune responses were suppressed by intestinal colonization with histamine-generating, DAG kinase-producing WT $L$. reuteri}

To identify whether $h d c A^{+} d g k A^{+} \operatorname{Lr}$ colonization had an impact on immunomodulation of the mouse intestine, proinflammatory cytokines including IL- $1 \alpha$, IL-6, tumor necrosis factor- $\alpha$, IL-17, IL-22, IL-12, eotaxin, granulocyte colony-stimulating factor (G-CSF), and interferon- $\gamma$ were analyzed in the blood plasma of gnotobiotic and GF control mice. Interestingly, mice colonized with WT $L$. reuteri yielded reduced circulating concentrations of IL- $1 \alpha$ compared with mice colonized by $L$. reuteri $\triangle d g k A$ or control GF mice 


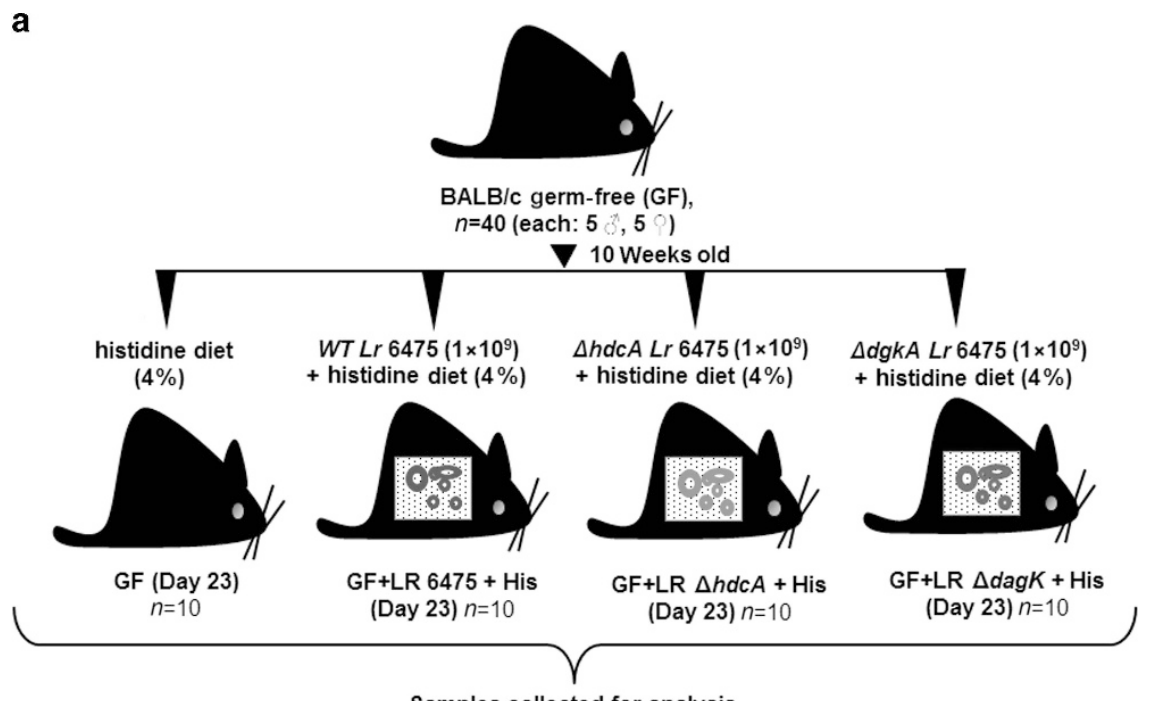

Samples collected for analysis

b

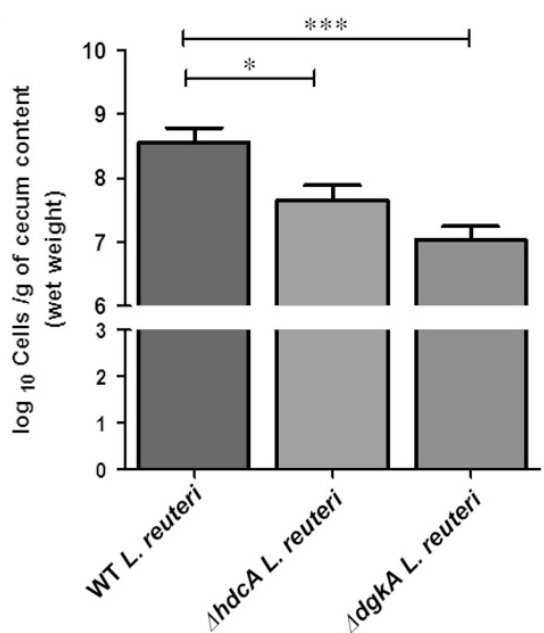

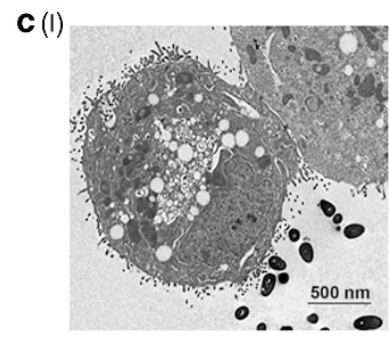

(II)

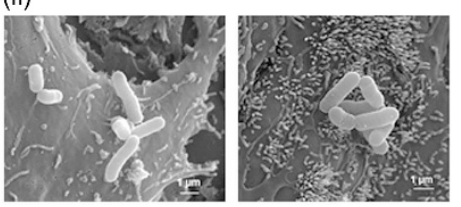

10 Wks, BALB/c GF mice

Figure 1 Colonization status of histamine-generating probiotic strain (Lactobacillus reuteri6475) in adult BALB/c mice. (a) BALB/c mice (10 weeks old) were colonized with $L$. reuteri ( $\mathrm{Lr}$ ) 6475 on day 1 and killed on day 23. The mice were fed with a histidine-rich (4\%) diet during the bacterial colonization period. Four groups differed by probiotic colonization status: group 1 germ-free (GF) control mice that lacked bacteria, group 2 mice received wild-type (WT) L. reuteri6475, group 3 received $\triangle h d c A$ L. reuteri6475, and group 4 received $\Delta d g k A L$. reuteri 6475 ( $n=10$ mice per group, 5 males and 5 females). (b) Cecal intestinal contents showed positive bacterial colonization of WT, $\triangle h d c A$, and $\triangle d g k A$ Lr after day 22 days of colonization. GF mice showed no amplification indicating the germ-free status. Data are expressed as mean \pm s.e.m. ${ }^{*} P<0.05$ and ${ }^{\star * \star} P<0.001 ; n=10$ mice per group. One-way analysis of variance with Bonferroni correction. (cl) Transmission electron microscopy or (cll) Scanning electron microscopy of HT29 cells co-cultured with WT Lr 6475 for (I) $2 \mathrm{~h}$ or (II) $6 \mathrm{~h}$ under anoxic condition. A full color version of this figure is available at the Mucosal Immunology journal online.

(Figure 2a). Similar differences were observed for the chemokines, eotaxin and G-CSF, in peripheral blood quantified in $\mathrm{BALB} / \mathrm{c}$ gnotobiotic mice colonized by WT L. reuteri and respective mutants (Figure $2 \mathbf{b}, \mathbf{c}$ ). In addition, circulating quantities of IL-6 were reduced in SW (outbred) mice that received WT L. reuteri (Supplementary Figure S2e) by orogastric gavage. In addition, amounts of plasma IL-10, a cytokine known to suppress inflammation, were increased in mice colonized by WT L. reuteri (Supplementary Figure S2f), whereas G-CSF and IL-6 were diminished (Supplementary Figure S2a,e) in SW mice colonized with WT L. reuteri (Supplementary Figure S2a-f).
Intestinal mucosal proinflammatory cytokine suppression by $h d c A^{+} d g k A^{+}$WT $L$. reuteri in gnotobiotic mice

Mucosal immune responses because of the presence and absence of histamine and/or diacylglycerol kinase of WT L. reuteri 6475 were measured in intestinal mucosal tissue samples. Cecal $I L-6$ mRNA in the mucosa was significantly decreased in mice colonized by WT $L$. reuteri as compared with mice colonized by mutant $\triangle d g k A L$. reuteri or control GF $\mathrm{BALB} / \mathrm{c}$ mice (Figure 2d). However, other cytokine mRNAs such as tumor necrosis factor- $\alpha$, interferon- $\gamma$, IL-17, IL-12, IL- $1 \alpha$, and IL- 22 were not altered or below the detection limit. Similarly, IL-6 mRNA and protein quantities were significantly 

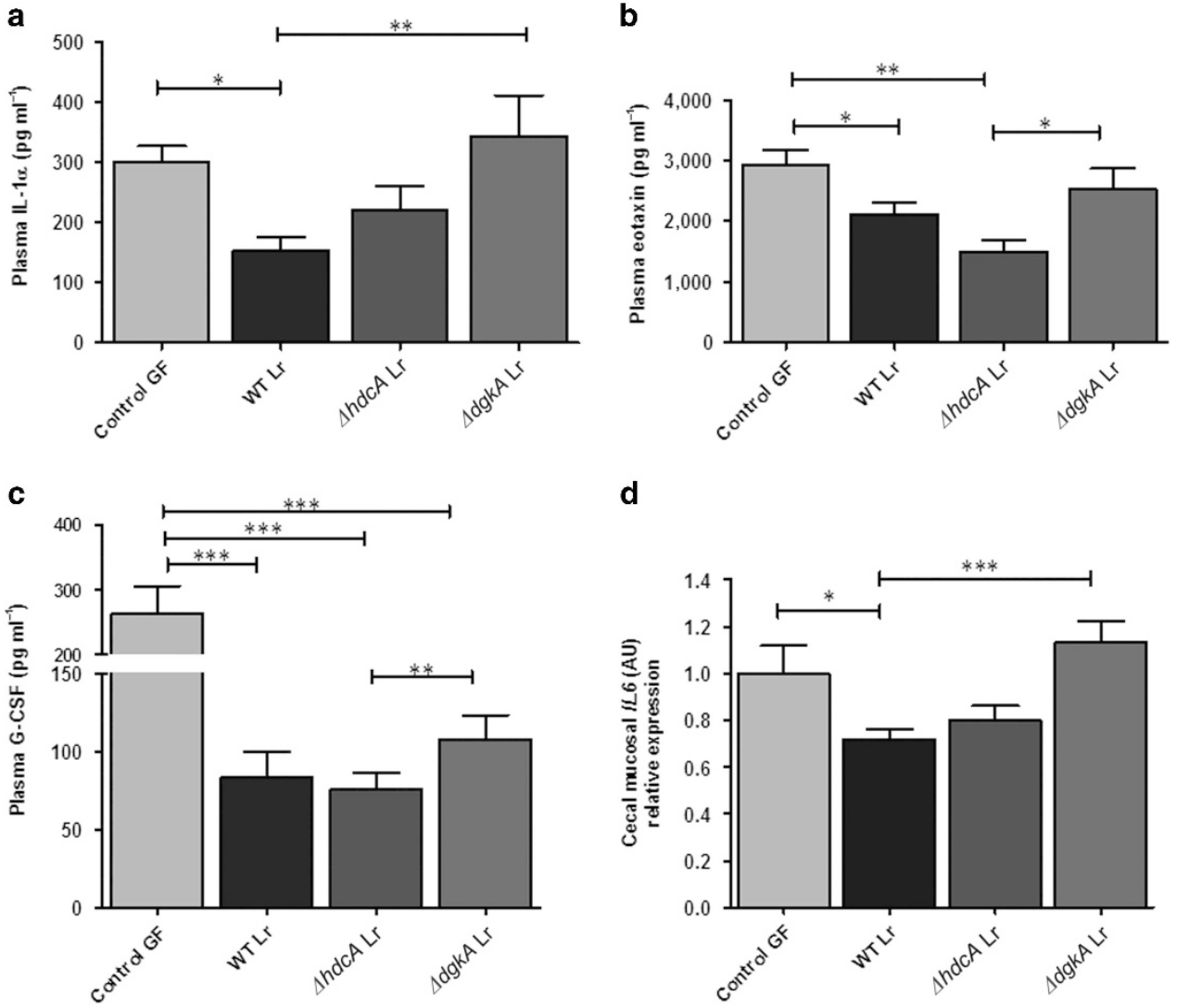

Figure 2 Lactobacillus reuteri colonization reduced proinflammatory responses in mono-associated BALB/c mice. BALB/c mice (10 weeks old) colonized with $L$. reuteri $(\mathrm{Lr}) 6475$ on day 1 and killed on day 23 showed reduced inflammatory biomarkers. MAGPIX multiplex MAP magnetic bead analysis on plasma of mice colonized with wild-type (WT), $\Delta h d c A$, and $\Delta d g k A$ Lr showing differences in protein levels of (a) interleukin (IL)-1 $\alpha$, (b) eosinophilic chemoattractant chemokine (eotaxin), (c) granulocyte colony-stimulating factor (G-CSF), and (d) cecum mucosal mRNA relative expression of IL6 by quantitative reverse transcriptase-PCR (qRT-PCR) analyzed in BALB/c mice colonized with WT, $\Delta h d c A$, and $\triangle d g k A L r$, as well as germ-free (GF) mice. Data are expressed as mean \pm s.e.m. ${ }^{\star} P<0.05$, ${ }^{\star \star} P<0.01$, and ${ }^{* \star} P<0.001 ; n=10$ mice per group. One-way analysis of variance with Bonferroni correction. A full color version of this figure is available at the Mucosal Immunology journal online.

reduced in the cecal mucosa of SW mice colonized by WT $L$. reuteri compared with control GF mice (Supplementary Figure S2g,i,j).

\section{Colonization by WT $d g k A^{+}$, but not mutant $\Delta d g k A$, L. reuteri inhibits mucosal PKC phosphorylation and NF-кB accumulation in intestinal epithelium}

As PKC phosphorylation depends on availability of DAG through GPCR activation, ${ }^{5}$ phosphorylation of pPKC was compared among the cecal mucosae of mice colonized by WT and mutant $\triangle h d c A$ or $\triangle d g k A$ L. reuteri, in addition to control GF mice. To determine that the bacterial enzyme Dgk, presumably released into the extracellular milieu, inhibits DAG-mediated signaling and PKC phosphorylation in mammalian cells, immunoblot studies were performed targeting PKC phosphorylation using specific antibodies. The phosphorylated protein kinase $\mathrm{pPKC} \alpha$ (Ser 657) was diminished in the cecal mucosa of gnotobiotic BALB/c mice colonized with WT Lr compared with control GF mice. In addition, BALB/c mice colonized by mutant $\triangle h d c A L$. reuteri had reduced amounts of pPKC when compared with control GF mice (Figure 3a). Interestingly, the greatest abundance of pPKC was localized in the intestines of mice colonized by mutant $\Delta d g k A$ L. reuteri as compared with GF mice colonized by WT L. reuteri or mutant $\triangle h d c A$ L. reuteri (Figure 3a,c). Consistent with the immunoblot data, pPKC was decreased in ceca of mice colonized by WT and mutant $\triangle h d c A$ L. reuteri when compared with mice colonized by mutant $\triangle d g k A$ L. reuteri (Figure 3b) evaluated by fluorescence immunohistochemistry. SW GF mice colonized by WT $L$. reuteri also showed reduced levels of pPKC compared with corresponding nonphosphorylated PKC and GF PKC protein concentrations from the cecal mucosa (Supplementary Figure S3a).

Human jejunal enteroids treated with WT L. reuteri conditioned media (CM) showed decreased pPKC levels compared with human enteroids treated with mutant $\triangle d g k A$ L. reuteri CM (Supplementary Figure S4a). In addition, $\Delta h d c A$ Lr CM-treated enteroids showed a trend toward increased PKC phosphorylation (Supplementary Figure S4a). Consistent with immunoblot data, pPKC was decreased in human enteroids treated with WT $L$. reuteri CM compared with mutant $\Delta d g k A \mathrm{Lr} C \mathrm{C}$, as examined by fluorescence immunohistochemistry (Supplementary Figure S4b). To explore mammalian intestinal epithelial DAG and pPKC signaling 

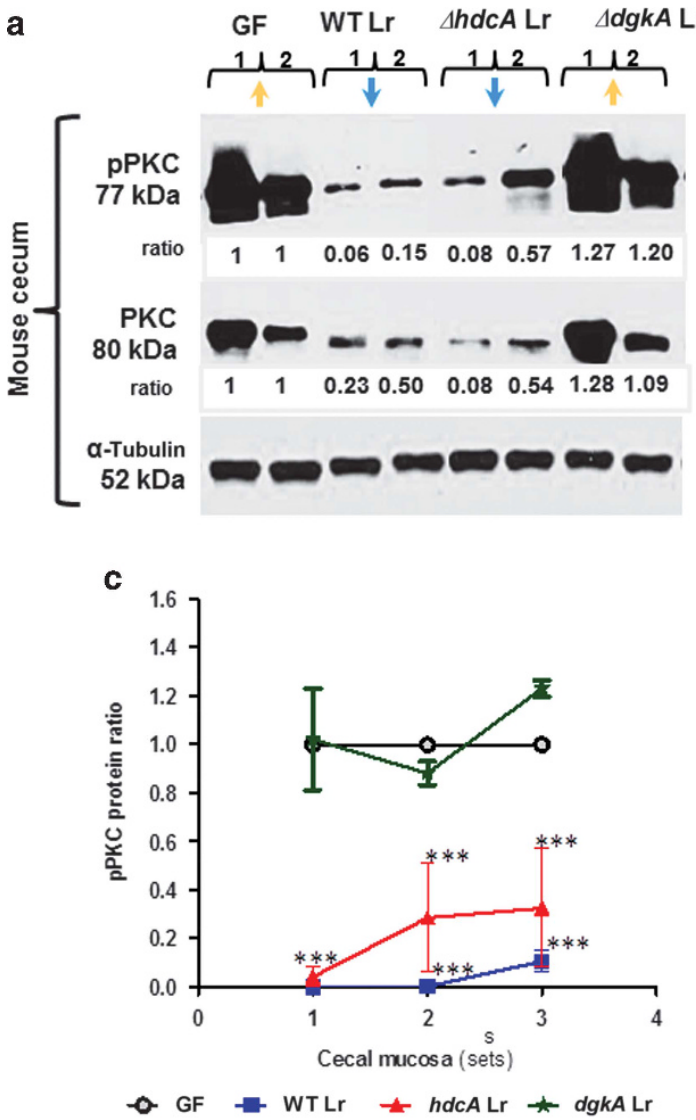

b
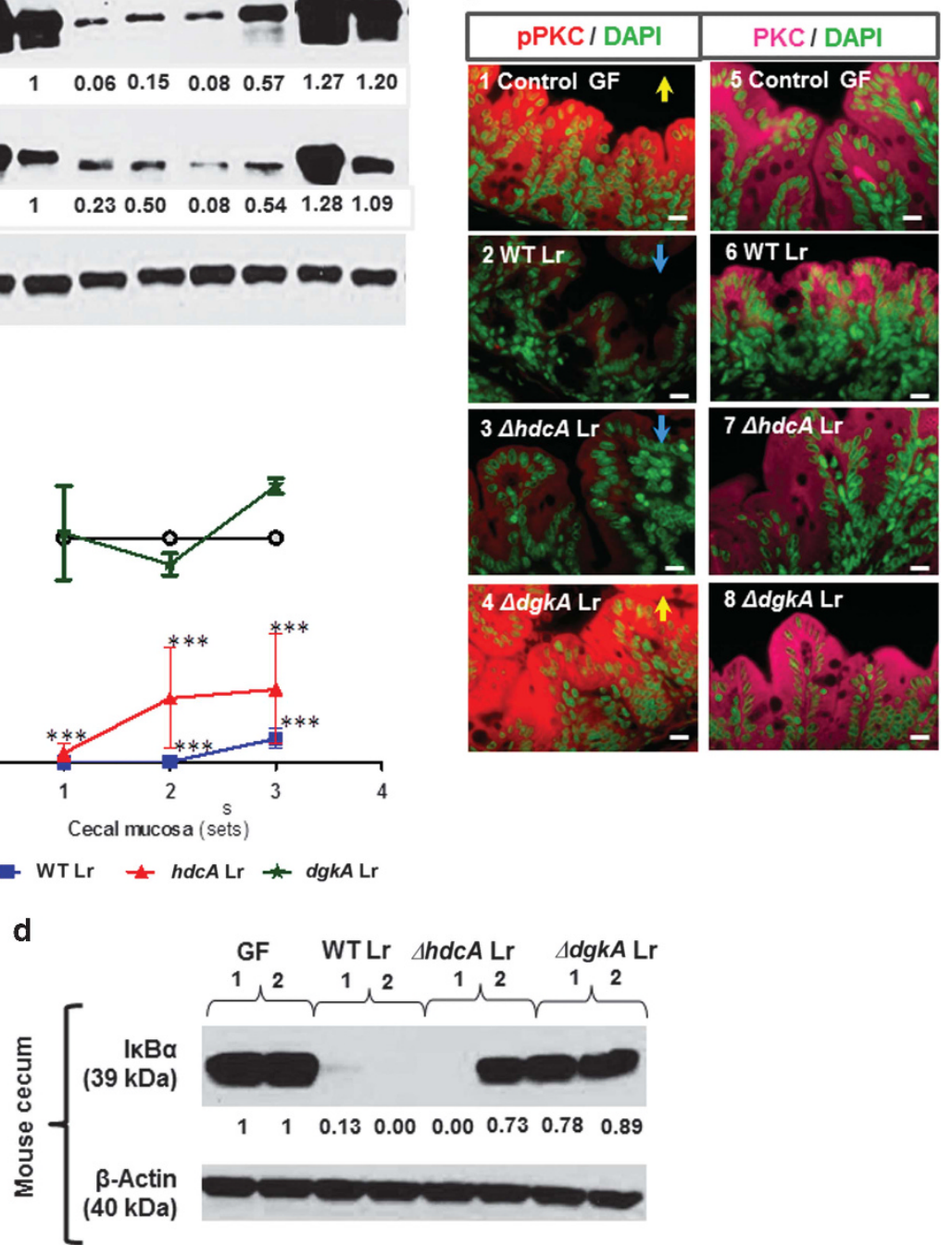

Figure 3 Lactobacillus reuteriderived diacylglycerol kinase (Dgk) synthesis prevents type 1 histamine receptor (H1R)-mediated protein kinase C (PKC) phosphorylation and nuclear factor (NF)- $\mathrm{kB}$ accumulation in mammalian intestinal epithelium. (a) BALB/c mice (10 weeks old) colonized with $L$. reuteri (Lr) 6475 on day 1 and killed on day 23 showed suppressed cecal pPKC. Western blot analysis of cecal mucosa proteins from wild-type (WT), $\triangle$ hdcA, and $\triangle d g k A$ Lr colonized mice and germ-free (GF) mice incubated with antibody targeting phospho-PKC (pPKC) or non-phospho-PKC (PKC). (1) Proteins pooled from all the five female mice and (2) protein pooled from all the five male mice per group. The proteins were normalized for housekeeping protein $\alpha$ tubulin. The pPKC and PKC ratio was obtained by image J analysis where pPKC and PKC from GF control mice was set to 1 and used as baseline. Then, $25 \mu \mathrm{g}$ of proteins were loaded into each wells of the sodium dodecyl sulfate (SDS) gel. Proteins were isolated from the pooled samples ( 1 to $5=1$ and 6 to $10=2$ ) 3 individual times and proteins were estimated by Bio-Rad analysis and western blot was performed for 3 individual times. (b) The pPKC and PKC analyzed using fluorescence immunohistochemistry from cecum tissue of BALB/c mice colonized with WT, $\triangle \mathrm{hdcA}$, and $\triangle d g k A L$. reuteri6475, and control GF mice. Red color indicates pPKC, pink color indicates PKC distribution of lamina propria, and green color indicates nuclei staining by 4',6-diamidino-2phenylindole (DAPI). Bar $=10 \mu \mathrm{m}$. Images shown are representative of five individual mouse sections per group. The proteins were normalized for housekeeping protein $\alpha$-tubulin. (a, b) Blue arrow indicates downregulation of $\mathrm{pPKC}$ and yellow arrow indicates upregulation of $\mathrm{pPKC}$. (c) Mean values of three independent western blot gels showing pPKC protein concentration (loaded $25 \mu \mathrm{g}$ ) and quantified by densitometry using imageJ. GF control groups were set to $1 .{ }^{\star \star \star} P<0.001 ; n=10$ mice per group. One-way analysis of variance with Bonferroni correction. (d) Western blot analysis of cecal mucosa

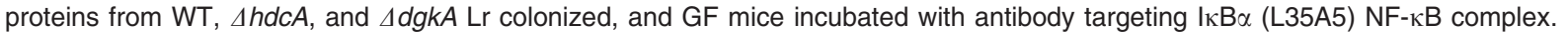

further, ileal enteroids from 10-week-old GF mice were treated with a DGK inhibitor in vitro. The enteroids treated with a DGK inhibitor (R59-022) yielded increased PKC phosphorylation in the presence of WT Lr CM, whereas enteroids lacking DGK inhibitor in the presence of WT Lr CM did not yield evidence of increase PKC phosphorylation (Supplementary Figure S5). Similarly, in vitro treatment of HT-29 human epithelial cells with $\triangle d g k A$ Lr CM yielded a nearly threefold increase in PKC phosphorylation compared with treatment with Lr WT CM (Supplementary Figure S4c). In addition, nonphosphorylated 
PKC protein concentrations increased over time in the presence of WT Lr CM and decreased with mutant $\triangle d g k A$ Lr CM treatment in HT-29 human epithelial cells (Supplementary Figure S4c).

Activation and accumulation of cytosolic nuclear factor $(\mathrm{NF})-\kappa \mathrm{B}(\mathrm{I} \kappa \mathrm{B} \alpha$-RelA:P50 complex) is dependent on phosphorylation of pPKC. ${ }^{37}$ The intestinal epithelial tissues obtained from the cecal mucosa of mice colonized by WT, mutant $\Delta h d c A$, or $\Delta d g k A$ L. reuteri were compared with control GF mice. To determine that the bacterial enzyme Dgk inhibits activation of NF- $\kappa \mathrm{B}$ through reduced phosphorylation of PKC in mammalian cells, immunoblot studies were performed targeting NF- $\kappa \mathrm{B}$ accumulation using specific antibodies. Total $\mathrm{I} \kappa \mathrm{B} \alpha$ (L35A5) complex was diminished in the cecal mucosa of gnotobiotic BALB/c mice colonized with WT Lr as compared with control GF mice. The $\triangle d g k A L$. reuteri colonized mice yielded increased synthesis of NF- $\kappa \mathrm{B}$ (Figure 3d).

\section{L. reuteri DAG kinase is secreted into the extracellular milieu}

As we observed negligible PKC phosphorylation in intestinal mucosal tissues of mice colonized with WT or $\Delta h d c A$ compared with $\triangle d g k A \operatorname{Lr}, \operatorname{Dgk}(\sim 15 \mathrm{kDa})^{11,38}$ is secreted by the bacterium Lr, and the extracellular microbial enzyme may effectively decrease DAG activity in mammalian cells. By suppressing DAG lipid-mediated signaling, DAG kinase from lactobacilli may suppress $\mathrm{H} 1 \mathrm{R}$ signaling via reduced $\mathrm{PKC}$ phosphorylation. To explore the possibility that bacterial Dgk may be secreted into the intestinal lumen and interact with substrate DAG in the host epithelium, Dgk was detected in bacterial culture supernatants following growth in liquid media. WT and mutant $h d c A$ or $\Delta d g k A$ L. reuteri 6475 were cultured in LDM4 for $12 \mathrm{~h}$ in the presence and absence of DAG, and liquid culture supernatants were evaluated.

Extracellular proteins were fractionated by size, processed for tryptic digestion, and analyzed by liquid chromatography/ tandem-mass spectrometry followed by amino acid sequence analysis. Amino acid sequences of Dgk peptide fragments (Supplementary Figure S6) matched the expected Dgk protein sequences (Figure 4a-c). As expected, Dgk was present in WT L. reuteri supernatants, but absent in mutant $\triangle d g k A$ L. reuteri cultures (Figure 4c). WT $\operatorname{Lr} d g k A$ mRNA levels were significantly increased in the presence of DAG compared with absence of DAG in the growth medium after $12 \mathrm{~h}$ in vitro (Figure 4d) and bacterial $d g k A$ gene expression was increased in cecal luminal contents of the gnotobiotic BALB/c mouse intestine colonized by WT $L$. reuteri (Figure 4f). In addition, clade II L. reuteri $6475 d g k A$ gene was present in greatest quantities during the elongation phase (Supplementary Figure S7). Bacterial mRNA was obtained from the cecal contents and quantified using reverse transcriptase-PCR. Bacterial $d g k A$ expression was increased in the lumens of both WT and $\Delta h d c A$ Lr colonized gnotobiotic BALB/c mice compared with $\triangle d g k A$ Lr colonized mice, whereas GF control BALB/c mice showed no evidence of $\operatorname{dgk} A$ expression (Figure 4f). In addition, mammalian DGK quantified from the cecal intestinal epithelium by immunoblot did not yield significant differences between control mice and the GF mice colonized with WT or mutant L. reuteri (Supplementary Figure S3b).

\section{Bacterial histidine decarboxylase mRNA and luminal histamine present in the intestinal lumen of gnotobiotic BALB/c mice colonized by $L$ reuteri}

Luminal contents were treated with $99 \%$ methanol and the supernatants obtained were used for histamine quantification using liquid chromatography/mass spectrometry. WT $L$. reuteri 6475 and mutant $\triangle d g k A L$. reuteri colonized BALB/ c mice yielded detectable luminal histamine (qualitative data not shown). Interestingly $\Delta d g k A \operatorname{Lr}$ culture grown for $24 \mathrm{~h}$ in lactobacillus defined media, LDM3 or LDM4, generated more than twofold increased histamine concentrations compared with WT Lr culture. Mutant $\Delta h d c A$ Lr yielded absence of detectable histamine in liquid media (Supplementary Table S1). However, mutant $\Delta h d c A$ Lr lacked detectable histamine in culture supernatants (Supplementary Table S1). Bacterial histidine decarboxylase mRNA, presumably at least partly from $L$. reuteri, was also detectable in the intestinal lumens of gnotobiotic BALB/c mice colonized by WT and mutant $\Delta d g k A$ L. reuteri (Figure $\mathbf{4 e}$ ).

\section{L. reuteri resides adjacent to the intestinal epithelium and modifies the intestinal mucus layer and GPCR signal of gnotobiotic mice}

Bacterial fluorescent in situ hybridization demonstrated that $\mathrm{Lr}$ resides in the mucus layer, penetrates the inner mucus layer, and establishes intimate contact with intestinal enterocytes in the murine large intestine (Figure 5a-f). The data suggest that Lr directly communicates with the host intestinal epithelium irrespective of WT or mutant ( $h d c A, d g k A$ ) status. Similarly, Lr was adjacent to the human epithelium when Lr was co-cultured with human jejunal enteroid monolayers (Figure 5g,h) and HT-29 cells (Figure 1c).

To determine H1R and H2R GPCR protein quantities, fluorescence immunohistochemistry analysis was performed as described in the Methods. Both $\mathrm{H} 1 \mathrm{R}$ and $\mathrm{H} 2 \mathrm{R}$ protein expression were similar between the WT, $\Delta h d c A$, and $\Delta d g k A$ Lr colonized mice compared with control GF mice (Figure 5i-p). However, H1R was present in greater abundance than $\mathrm{H} 2 \mathrm{R}$ in the intestinal epithelium.

Mucus serves as the primary contact between the intestinal epithelial layer and luminal antigens. ${ }^{39,40}$ As GF mice were colonized with Lr, it was of interest to evaluate mucus maturation after probiotic colonization using lectin staining. We observed significantly increased mucus fucosylation with Ulex europaeus agglutinin staining of the terminal mucus fucose in WT Lr colonized compared with control BALB/c GF mice (Supplementary Figure S8ai and aii). Similarly, increased fucosylation was observed in mice colonized with $\triangle h d c A$ and $\Delta d g k A$ Lr compared with control BALB/c GF mice. However, $\triangle d g k A$ colonized mice had decreased mucus fucosylation compared with WT Lr colonized mice (Supplementary Figure S8ai-aiv). Similarly, SW mice colonized with WT Lr also showed increased mucus fucosylation with increased 
a

1 MDSRDKHQTE KNHHLIQAMC HAIDGIIQVL REERNMRYHL LAACLAIIMS ILLHISAMEW LWILLAIFVV FTSEFLNTVT 3
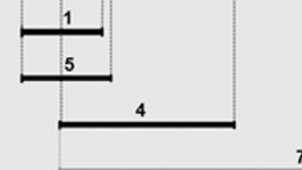

81 EAVTDLIVDH HYELNVKKAK DVAAGGVLIS AIFSVLVGLI IFIPRILAII $R$

6

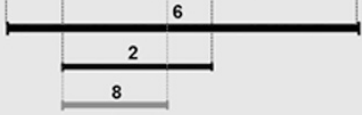

b

\begin{tabular}{|c|c|c|c|c|}
\hline No & Protein accession & Dgk peptide $(20 \mathrm{kDa})$ with DAG & Mass & Length \\
\hline 1 & gi|324978333|gb|EGC15283.1] & EERNMR & 833.38 & 6 \\
\hline 2 & gi|324978333|gb|EGC15283.1| & DVAAGGVLISA & 971.53 & 11 \\
\hline 3 & gi|324978333|gb|EGC15283.1| & DKHQTEK & 884.44 & 7 \\
\hline 4 & gi|324978333|gb|EGC15283.1| & NMRYHLLAACLAI & $1,487.77$ & 13 \\
\hline 5 & gi|324978333|gb|EGC15283.1| & EERNMRY & 996.44 & 7 \\
\hline 6 & gi|324978333|gb|EGC15283.1] & KAKDVAAGGVLISAIFSVLVGLIIFIP & $2,710.64$ & 27 \\
\hline No & Protein accession & gk peptide $(20 \mathrm{kDa})$ without DAG & Mass & Length \\
\hline 7 & gi|324978333|gb|EGC15283.1] & NMRYHLLAACLAIIMSILLHISAMEWLWILLAIFVVFTS & $4,515.45$ & 39 \\
\hline 8 & gi|324978333|gb|EGC15283.1] & DVAAGGVL & 700.38 & 8 \\
\hline
\end{tabular}

C

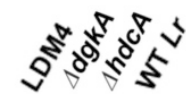

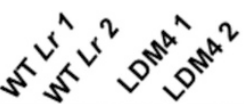

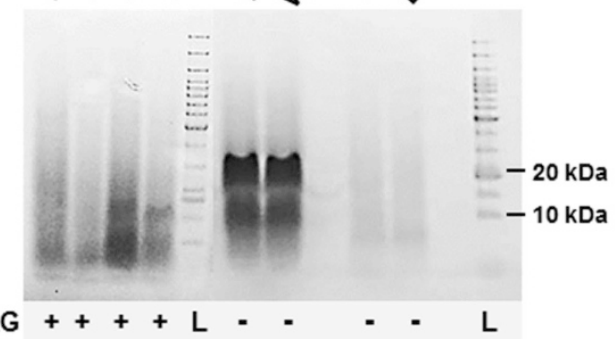

DAG

L

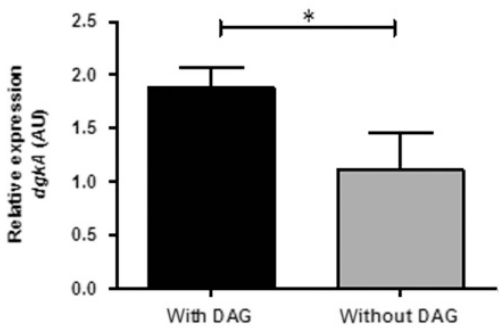

LDN4 $12 \mathrm{~h}$ culture

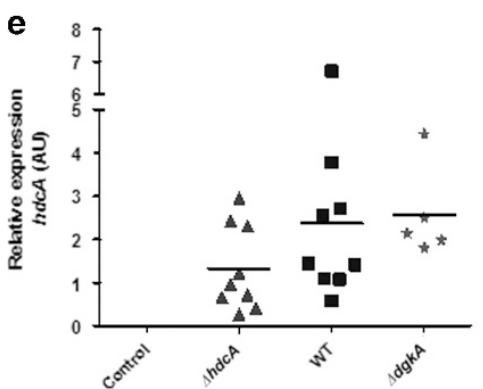

f

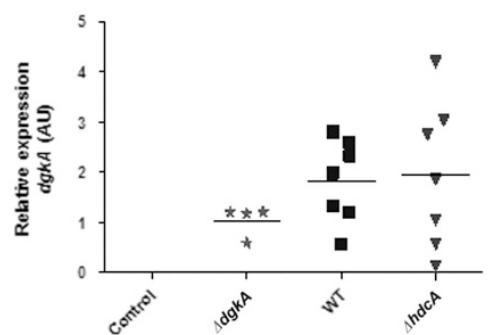

Figure 4 Lactobacillus reuteri 6475 derived diacylglycerol kinase (Dgk) secreted into the medium. (a) Conditioned media (CM) from wild-type (WT) $L$. reuteri ( $L r) 6475$ grown in LDM4 with lipid diacylglycerol (DAG) supplements for $12 \mathrm{~h}$ were analyzed using Ekisgent nanoLC and the $A B C I E X$ TripleTOF 5600 mass spectrometer. The obtained sequences were matched with the NCBI database with the Analyst software version 1.6 (ABCIEX). Red line indicates the protein sequence matching $L$. reuteri Dgk protein in the database from medium grown in the presence of lipid DAG, whereas blue line indicates the protein sequence matching $L$. reuteri Dgk in the database from medium grown in the absence of lipid DAG. The protein sequences were obtained from $20 \mathrm{kDa}$ band (also (c)). (b) CM from WT, $\Delta \mathrm{hdcA}$, and $\triangle d g k A \mathrm{Lr} 6475$ grown in LDM4 without lipid DAG supplement for $12 \mathrm{~h}$ were analyzed as mentioned in a. (c) One-dimensional sodium dodecyl sulfate (SDS) gel showing positive bands at 20 and $10 \mathrm{kDa}$ from L. reuteri derived CM with and without DAG supplementation. (a-c) The $20 \mathrm{kDa}$ protein band was cut and followed by tryptic digestion and were used to analyze as mentioned in $\mathbf{a}$ and $\mathbf{b}$. (d) mRNA expression of $d g k A$ in WT L. reutericultured for $12 \mathrm{~h}$ with and without lipid DAG supplementation in LDM 4 minimal media in $\mathrm{N}_{2} / \mathrm{CO}_{2}$ at $37^{\circ} \mathrm{C}$ and analyzed by reverse transcriptase-PCR (RT-PCR). All experiments were repeated 3 times individually; $n=3$ per group. Data are expressed as mean \pm s.e.m. ${ }^{*} P<0.05$, one-way analysis of variance with Bonferroni correction. Graph showing cecal bacterial (e) $h d c A$ and (f) $d g k A$ gene expression levels of BALB/c mice colonized with WT, $\triangle h d c A, \Delta d g k A L r$, and germ-free (GF) control. (e, f) Data are expressed as median with range. ${ }^{*} P<0.05 ; n=10$ mice per group. Kruskal-Wallis test with Dunn's correction. A full color version of this figure is available at the Mucosal Immunology journal online. 


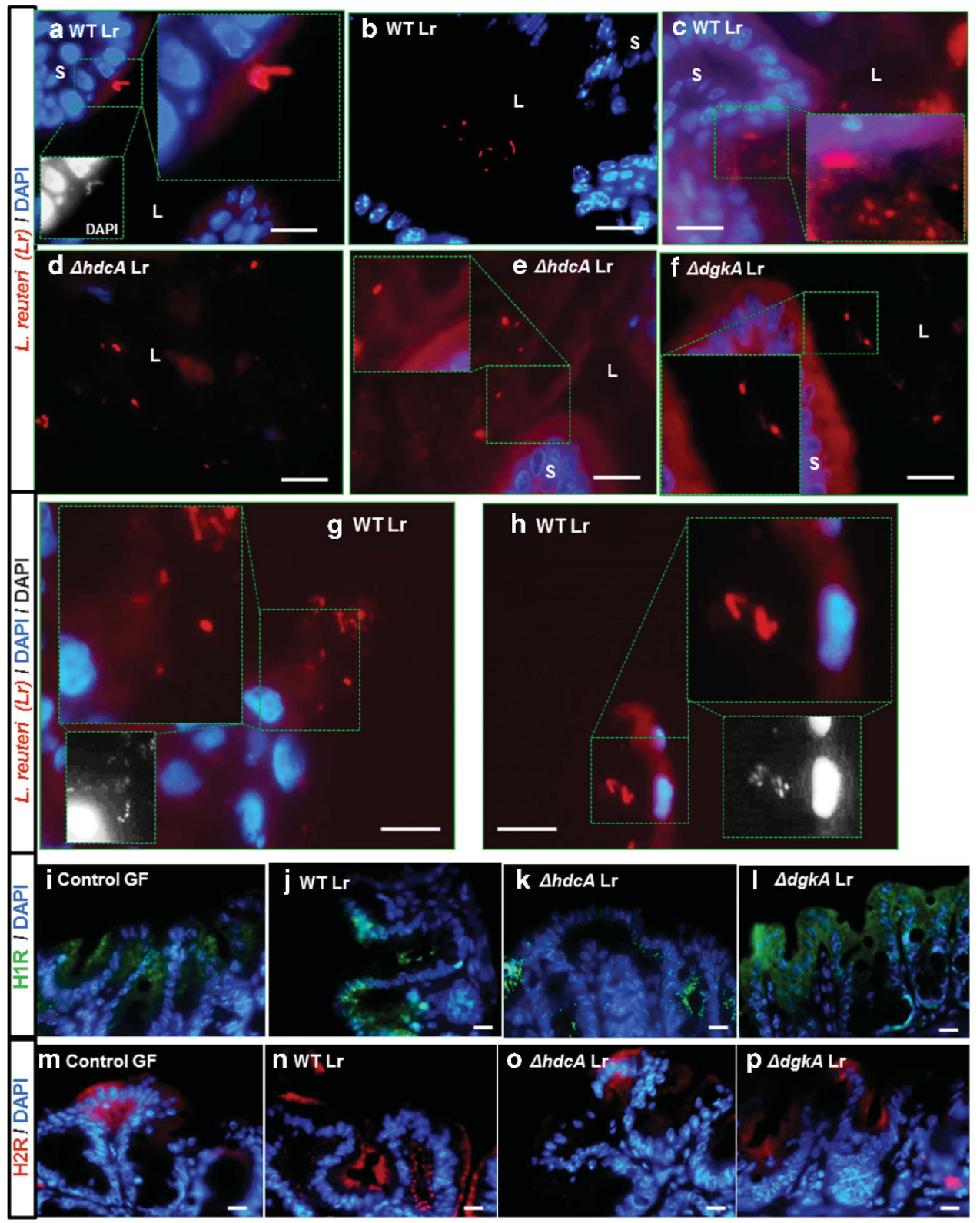

Figure 5. Lactobacillus reuteri resides close to intestinal epithelium and epithelial histamine receptors. Fluorescent in situ hybridization (FISH) analysis used to see $L$. reuteri $(\mathrm{Lr}$ ) localization in the mouse intestine (a-c) wild-type (WT), (d, e) $\Delta h d c A$, and (f) $\Delta d g k A$ Lr 6475 . Red color indicates the bacterium and blue color (or) white color indicates the nuclei stained with 4',6-diamidino-2-phenylindole (DAPI). Original magnification $\times 100$. Representative images of six mice per group. Bar $=200$ pixels. (g, h) WT L. reuteri 6475 interaction on the human jejunal enteroids monolayer visualized by FISH. Red color indicates the bacterium and blue color (or) white color indicates the nuclei stained with DAPI. Original magnification $\times 100$. Bar $=200$ pixels. L, luminal; S, serosal side. Histamine receptor 1 (H1R) analyzed using fluorescence immunohistochemistry from cecum tissue of 10-week-old (i) germfree (GF) BALB/c mice colonized with (j) WT, (k) $\triangle h d c A$, (I) and $\triangle d g k A$ Lr 6475. Green color indicates H1R distribution of cecal epithelium and blue color indicates nuclei by DAPI staining. Histamine receptor $2(\mathrm{H} 2 \mathrm{R})$ analyzed using fluorescence immunohistochemistry from cecum tissue of 13-week-old (m) GF BALB/c mice colonized with (n) WT, (o) $\Delta h d c A$, and (p) $\Delta d g k A$ L. reuteri 6475 . Red color indicates H2R distribution and blue color shows nuclei by DAPI staining. (i-p) Original magnification $\times 60$. Bar $=10 \mu \mathrm{m}$. Representative images of six mice per group.

mucus secreting goblet cells (Supplementary Figure S8av-aviii and $8 b$ ).

\section{Histamine-dependent enhancement of CD11b ${ }^{+} \mathrm{Gr}-1^{+}$ Ly6G ${ }^{\text {high }}$ splenic myeloid cell maturation was offset by the absence of DAG kinase in $L$. reuteri}

To determine whether bacterial-derived histamine has immunomodulatory effects by targeting myeloid cell maturation, GF mice were colonized with WT, $\Delta h d c A, \Delta d g k A \mathrm{Lr}$, and a control group without any bacteria. The absence of endogenous histamine results in increased proportions of IMCs and has been associated with cancer progression in mammals. ${ }^{14}$ Azoxymethane/dextran sodium sulfate-treated $\mathrm{Hdc}^{-/-}$male mice showed significantly reduced $\mathrm{CD}_{11 \mathrm{~b}}{ }^{+} \mathrm{Gr}-1^{+}$IMCs (80\%) in spleens after oral administration of $h d c^{+} \operatorname{Lr} 6475$ (WT) treatment. ${ }^{41}$ In this study, BALB/c gnotobiotic mice 
a

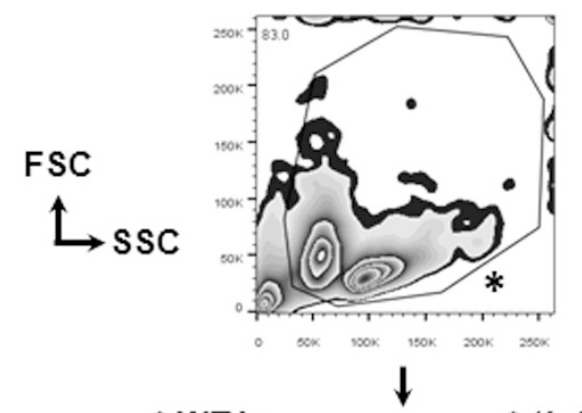

${ }^{*}$ Control GF

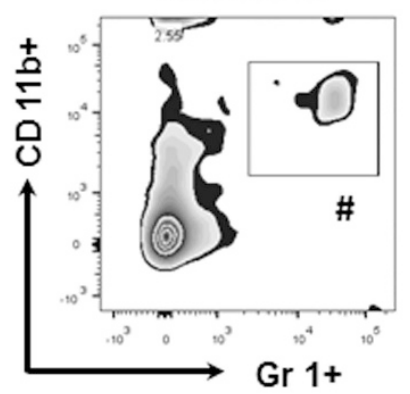

${ }^{*}$ WT Lr

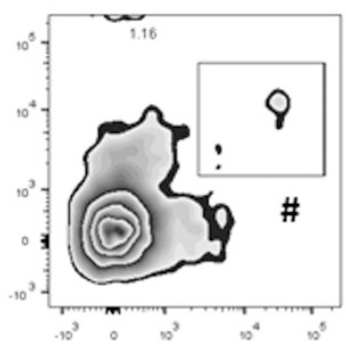

${ }^{*} \Delta h d c A \mathrm{Lr}$

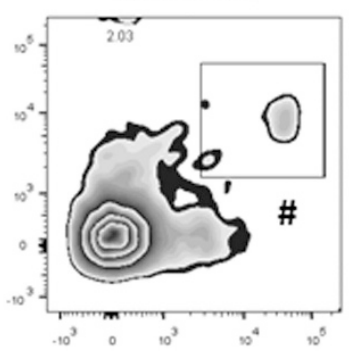

* $\Delta d g k A \mathrm{Lr}$

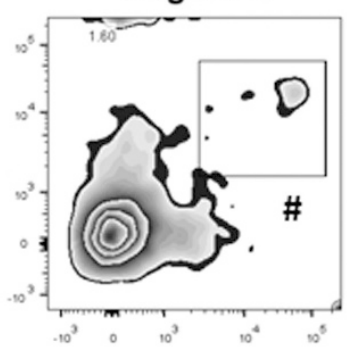

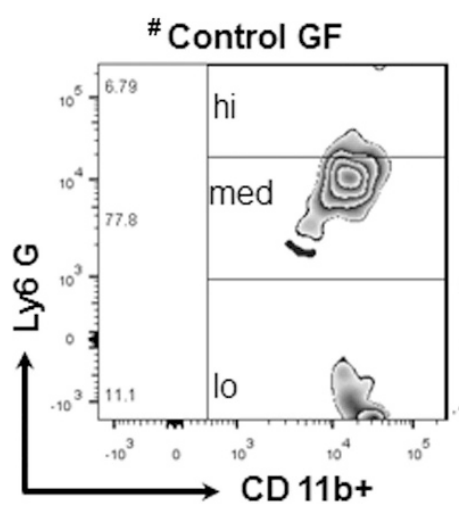

b

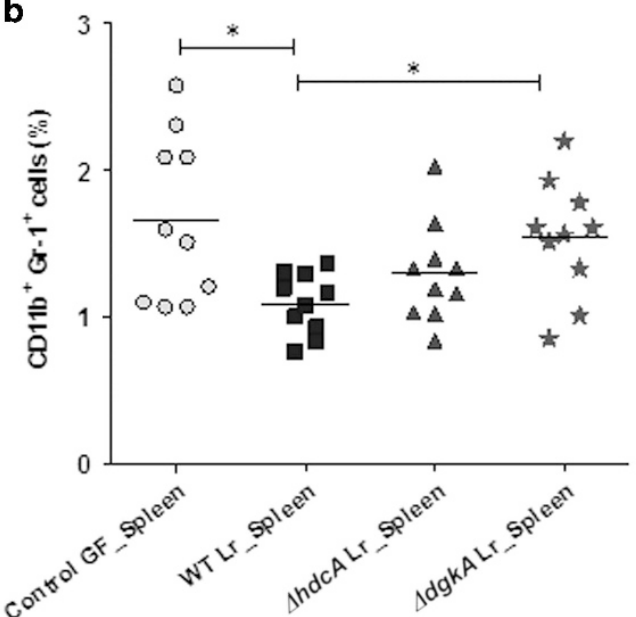

\# $\Delta h d c A \mathrm{Lr}$

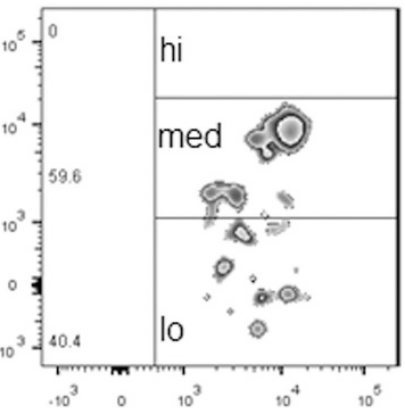

${ }^{\#}$ WT Lr

\section{$\downarrow$}
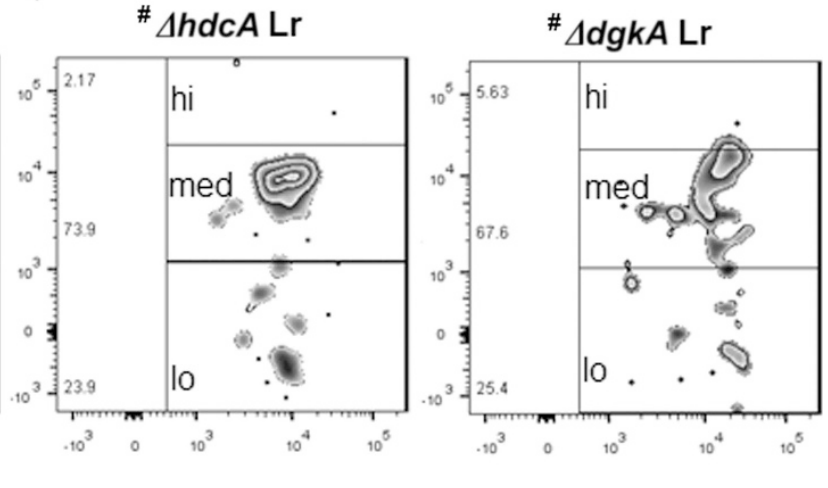

c

\begin{tabular}{|c|c|}
\hline \multicolumn{2}{|c|}{ Cecum tissue } \\
\hline mmu-miR-669k-3p & Fold change \\
\hline Control GF & 1.00 \\
\hline WT Lr & $2.0057^{\text {*x }}$ \\
\hline$\Delta h d c A ~ L r$ & 1.26 \\
\hline$\Delta d g k A ~ L r$ & 0.879 \\
\hline
\end{tabular}

Figure 6 Lactobacillus reuteri colonization reduces splenic immature myeloid cells (IMCs) with increased cecal mmu-miR-669k-3p. BALB/c mice (10 weeks old) were colonized with wild-type (WT), $\Delta$ hdcA, $\Delta d g k A$ L. reuteri (Lr) 6475, and germ-free (GF) control. (a) Zebra plot showing CD11b ${ }^{+}$Gr1 ${ }^{+}\left(\mathbf{a}^{*}\right)$ IMCs with ( $\left.\mathbf{a}^{\#}\right)$ Ly6G ${ }^{\text {hi }}$, Ly6G ${ }^{\text {med }}$, and Ly6G ${ }^{\text {lo }}$ in spleen of mice. Representative images of $n=10$ mice per group; hi, high, lo, low; med, medium. (b) Splenic IMCs of WT, $\Delta h d c A$, and $\Delta d g k A$ Lr colonized, and control GF mice. Data are expressed as mean \pm s.e.m. ${ }^{\star} P<0.05 ; n=10$ mice per group. One-way analysis of variance with Bonferroni correction. (c) Table shows microRNA (miRNA) data obtained from cecum mucosa of WT, $\Delta$ hdcA, and $\triangle d g k A$ Lr colonized, and control GF mice quantified using ViiA7 real-time reverse transcriptase-PCR (rtRT-PCR). From 84 miRNAs quantified, one miRNA, mmumiR-669k-3p $\left(^{* *}\right)$, showed significant increase. Each group represents cecum mucosal miRNA pooled from 10 mice. Significant differences and $\Delta$ Ct were obtained by web-based BmiRNA PCR Array Data Analysis software (QIAGEN). A full color version of this figure is available at the Mucosal Immunology journal online. 
colonized with WT $L$. reuteri strain yielded significantly decreased proportions of $\mathrm{CD}_{11 \mathrm{~b}^{+} \mathrm{Gr}-1^{+}}$IMCs in the spleen (Figure 6b), suggesting that histamine-generating Lr promotes maturation of splenic CD11b ${ }^{+} \mathrm{Gr}-1^{+}$IMCs (Figure 6a,b). The lack of bacterial histidine decarboxylase in $\Delta h d c A \mathrm{Lr}$ corresponded with a trend toward increased populations of IMCs in the spleen (Figure 6b). In addition, GF mice colonized with $\triangle d g k A$ Lr did not result in suppression of IMCs in the spleen (Figure 6b), suggesting that loss of microbiome-derived DAG kinase and enhanced H1R signaling may offset effects via $\mathrm{H} 2 \mathrm{R}$ on myeloid cell maturation. In addition, we observed reduced $\mathrm{Ly} 6 \mathrm{G}^{\text {high }}$ and Ly $6 \mathrm{G}^{\text {medium }}$ populations in the spleens of mice that were colonized with WT $L$. reuteri as compared with mice colonized by mutant $\triangle h d c A$ or $\triangle d g k A L$. reuteri (Figure 6a).

\section{L. reuteri colonization leads to increased microRNA mmu- miR-669k-3p in the intestinal epithelium of GF BALB/c mice}

Because basal cytokine responses were suppressed in the presence of WT L. reuteri in the intestines of gnotobiotic BALB/ c mice (Figure 2), changes in inflammatory responses were related to microRNA modification. A total of 84 individual microRNAs considered as candidates for regulating inflammatory pathways were selected. The microRNA expression patterns in the intestinal mucosa were evaluated for alterations by Lr-derived histamine and/or Dgk. The miRNA mmu-miR-669k-3p was found to be increased by twofold in mice colonized with WT Lr compared with $\Delta h d c A$, $\Delta d g k A \mathrm{Lr}$, and control GF mice (Figure 6d).

\section{DISCUSSION}

The mammalian microbiome contains microbial cells with genomes encoding the capacity for microbial-mediated conversion of amino acids to bioactive signals such as biogenic amines. ${ }^{17}$ As shown in this study, bacterial enzymes such as diacylglycerol kinase may abrogate mammalian cell signaling pathways by effectively "removing" mammalian lipid signals such as diacylglycerol. The gut commensal microbe, L. reuteri, secretes Dgk and because of its close proximity to the gut epithelium, this enzyme may convert mammalian DAG to PA. The presence of dgkA-producing $L$. reuteri suppresses phosphorylation of PKC downstream of the histamine type 1 receptor. Dgk secreted by Lr 6475 potentially interacts with epithelial DAG lipid signaling, thereby inhibiting proinflammatory H1R and allowing "net positive" activation of H2R by histamine-generating Lr. Ultimately, this reduced phosphorylation of PKC may result in diminished signaling via $\mathrm{NF}-\kappa \mathrm{B}$ and suppression of mucosal inflammation.

Human-derived clade II L. reuteri strains contain a complete chromosomal $h d c$ gene cluster with genes encoding histidine decarboxylase (HdcA) and the histidine/histamine transporter (HdcP), ${ }^{19}$ culminating in suppression of human tumor necrosis factor production. ${ }^{17}$ Gut microbes like L. reuteri clade II strains provide a useful model system to study the effects of luminal histamine on the mammalian gut mucosa. By introducing individual gut microbes into GF mice, our data show that WT and mutant $L$. reuteri each colonized the mammalian intestine successfully with a single oral dosing. Although immunologists have accumulated insights regarding how histamine works in allergic inflammation ${ }^{42}$ and in the context of mucosal mast cells, ${ }^{12}$ the role of histamine in the lumen of the gastrointestinal tract is not well understood. The effects of luminal histamine may differ from that of mucosal histamine simply based on the relative distributions of histamine receptors in different cell types. Our studies suggest that luminal histamine does affect mucosal and systemic immunity, as well as mammalian epithelial cell signaling pathways. Suppression of IL-6 production within the cecal mucosa is one example of histaminedependent suppression of gut inflammation in an acute colitis model. ${ }^{34}$ By investigating the role of luminal histamine produced by gut microbes, we may gain a deeper mechanistic understanding of the interrelationships between the gut microbiome, the intestinal mucosa, and immunity.

Luminal histamine may act through the histamine type 2 receptors (to suppress the production of proinflammatory cytokines $^{30}$ and cell signaling via mitogen-activated protein kinases (see Thomas et al. ${ }^{17}$ and Gao et al. ${ }^{34}$ ). However, both $\mathrm{H} 1 \mathrm{R}$ and $\mathrm{H} 2 \mathrm{R}$ are present in the mouse and human intestinal epithelium, and hence the role of $\mathrm{H} 1 \mathrm{R}$ in promoting gastrointestinal mucosal inflammation must be considered. ${ }^{5}$ Our studies provide insights into a potential "brake" directed at H1R-mediated signaling. A bacterial enzyme, Dgk, may modify the lipid signal diacylglycerol to abrogate $\mathrm{H} 1$ receptor-mediated signaling via $\mathrm{PKC}$. In contrast, histamine binding to $\mathrm{H} 2 \mathrm{R}$ activates protein kinase $\mathrm{A}$ and binds cAMP via adenylate cyclase $^{43}$ to suppress mitogen-activated protein kinase activation. Instead, H1R signaling involves the conversion of phosphatidylinositol 2 by phospholipase C into DAG and inositol triphosphate. ${ }^{25,43,44}$ DAG stimulates phosphorylation of $\mathrm{PKC}^{5,23,44}$ by binding to the catalytic domain 1 and recruiting PKC to the membrane. The interaction of PKC with the membrane causes release of pseudosubstrate from the catalytic domain leading to availability of serine and threonine phosphoacceptor residues causing PKC activation. ${ }^{45}$ Phos- $^{-}$ phorylated $\mathrm{PKC} \alpha(\mathrm{pPKC})$ is known to induce proinflammatory responses such as NF- $\kappa \mathrm{B}$ activation. ${ }^{43,46}$ Thus, a net reduction of $\mathrm{pPKC}$ in the intestinal mucosa by the gut microbiome may help to keep the immune system "in check" and promote immunologic homeostasis.

Beneficial microbes in the intestine may produce enzymes that inhibit mammalian cell signaling pathways by effective signal depletion. DGK is an enzyme known to inhibit DAGmediated signaling by converting DAG to (PA utilizing adenosine triphosphate as the phosphate source. ${ }^{3,47-49}$ Conversion of DAG to PA depletes DAG, and this reduced amount of DAG may suppress PKC activity, ${ }^{47,49}$ thereby suppressing proinflammatory immune responses in the intestine (Figure 7). On that note, Escherichia coli has increased amounts of DAG kinase. Resting human platelets $\left(10^{9}\right.$ cells have $0.111 \mathrm{nmol}$ DAG) treated with $E$. coli DAG kinase results in conversion of lipid DAG to PA. ${ }^{50}$ We propose that the mammalian microbiome may synthesize Dgk adjacent to the 


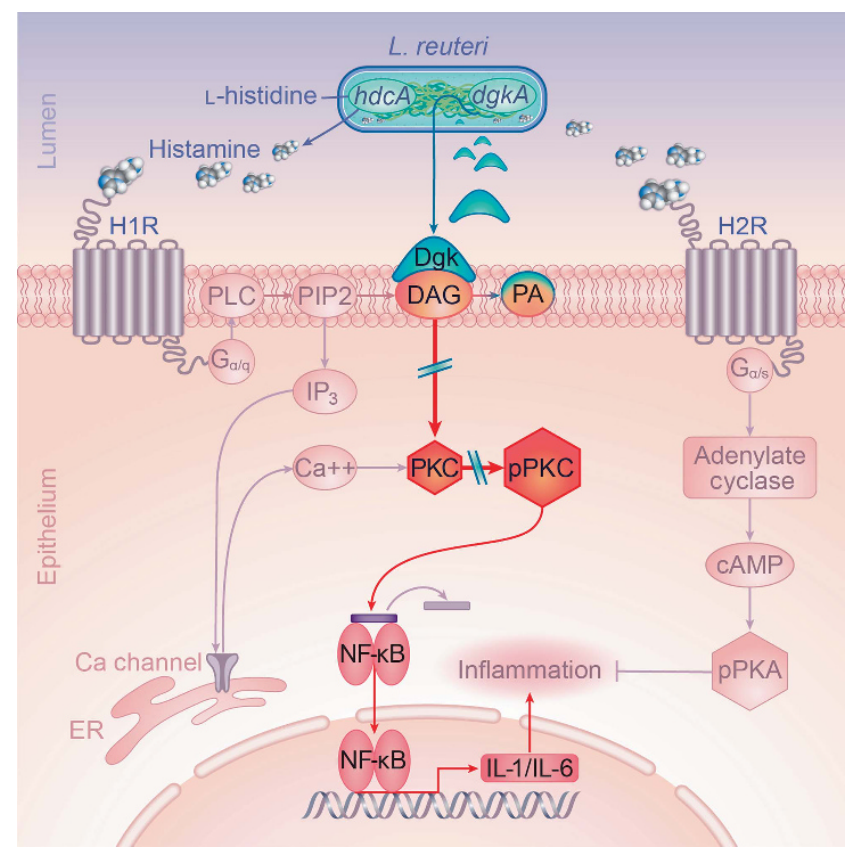

Figure 7 Schematic description of Lactobacillus reuteri derived metabolite signaling through intestinal epithelium in mammals. Histamine secreted by Lactobacillus reuteri 6475 activates $\mathrm{G}$ protein-coupled histamine receptors $\mathrm{H} 1$ and $\mathrm{H} 2$. Diacylglycerol kinase (Dgk) synthesized by $L$. reuteri inhibits epithelial $\mathrm{H} 1 \mathrm{R}$ downstream signaling and reduces protein kinase $\mathrm{C}$ (PKC) phosphorylation with decreased proinflammatory biomarkers. DAG, diacylglycerol; Dgk, diacylglycerol kinase; ER, endoplasmic reticulum; Hdc, histidine decarboxylase; HR, histamine receptor; $\mathrm{IP}_{3}$, inositol triphosphate; $\mathrm{PA}$, phosphatidic acid; PIP2, phosphatidylinositol 2; PK, phosphorylated protein kinase (C or A); PLC, phospholipase C.

intestinal epithelium, effectively depleting mammalian DAG and inhibiting H1R downstream activation. In support of our proposed hypothesis, L. reuteri is positioned adjacent to the intestinal epithelium and could deliver DAG kinase in close proximity to the epithelial cell membranes. PKC phosphorylation was drastically reduced in the intestinal epithelium of WT compared with $\triangle d g k A$ Lr colonized mice. Dramatically increased phosphorylation of PKC was evident in the intestinal mucosa of mice colonized by $\triangle d g k A \mathrm{Lr}$ and control GF BALB/c mice, but not in mice colonized by WT or $\triangle h d c A$ L. reuteri. Increased PKC phosphorylation was observed in human cells such as the human intestinal epithelial cell line HT29 treated with $\triangle d g k A \mathrm{Lr}-\mathrm{CM}$. These findings raise intriguing possibilities of bacterial enzymes from the mammalian microbiome converting compounds within the mammalian cell milieu and altering signaling pathways in mammalian cells. Mammalian epithelial cells may, for brief periods of time, behave like "chimeric" cells with bacterial and mammalian enzymes affecting intra-mammalian cell signaling pathways.

Previous findings showed that bacterial Dgk shares 18 to $19 \%$ amino acid sequence identity with human $\mathrm{DGK}^{8}$ that can have soluble isoforms. ${ }^{8,11,38}$ Similarly, Staphylococcus aureus DAG kinase B structure has been identified to be directly relevant to the human enzymes that function in cell signaling because the key structural and active site residues are conserved in the sequences of both enzymes. ${ }^{8}$ The bacterial Dgk superfamily provides candidate enzymes and substrates for evaluation of small-molecule therapeutics to combat Gram-positive organisms and to modulate lipid signaling pathways in humans. ${ }^{8}$ Mammalian DGK is found both in soluble and membranebound forms actively converting lipid DAG to PA, ${ }^{51}$ thereby suppressing PKC phosphorylation via DAG phosphorylation to PA. $^{52}$ We hypothesized that Dgk released by Dgk-positive intestinal lactobacilli can suppress H1R downstream signaling by modifying mammalian cell membrane-associated DAG. It is not understood whether a bacterial kinase from the microbiome may directly convert membrane-associated DAG in adjacent mammalian epithelial cells. We present evidence that downstream mammalian PKC is activated in the absence of gut microbes, but suppressed in the presence of histamine-generating microbes with an intact DAG kinase gene. In support of the hypothesis, Lrderived Dgk could not suppress intestinal epithelial PKC phosphorylation in the presence of a DGK inhibitor.

Prior evidence indicates that bacterial DAG kinase may be secreted or released by microbes into the extracellular milieu. ${ }^{8,50}$ Prior studies have also indicated that mammalian DAG kinases may be released; ${ }^{51}$ however, mammalian DAG kinase was not significantly altered by the presence of $L$. reuteri DAG kinase in gnotobiotic mice. DAG kinases bind to lipid DAG and generate PA in the lipid bilayer and these compounds may be available for effects on intracellular signaling pathways. Bacillus subtilis has been shown to produce $34 \mathrm{kDa}$ soluble Dgk enzyme. ${ }^{11}$ We looked for soluble Dgk proteins/peptides in the supernatant obtained from WT Lr culture for $12 \mathrm{~h}$ in LDM4. By liquid chromatography/tandem-mass spectrometry analysis we generated the presence of six peptide sequences from bacterial culture supernatants, and these peptide sequences were identical to segments of the Dgk protein (http:// www.ncbi.nlm.nih.gov/protein/EGC15283.1) from Lr 6475. In addition, $\operatorname{Lr} 6475$ was found to colonize the germ-free mouse intestine, adjacent to the intestinal epithelium. L. reuteri is known to both possess secretory and extracellular proteins (secretome) and these secreted proteins include enzymes involved in lipid metabolism. ${ }^{53,54}$ DAG is a well-known mammalian membrane lipid compound that flip-flops rapidly in the lipid bilayer. ${ }^{55}$ Dgk secreted by L. reuteri may interact with host DAG in the gut epithelium, reducing amounts of DAG and thereby restricting PKC phosphorylation and NF- $\kappa B$ activation $^{37}$ following downregulation of mucosal cytokines like IL-6. Such a reduction in mucosal inflammation or dampening of mucosal immunity may result in altered maturation programs of splenic myeloid cells. ${ }^{14}$

A deficiency HDC and lack of endogenous histamine was shown to promote inflammation-associated colorectal cancer by accumulation of $\mathrm{CD} 11 \mathrm{~b}^{+} \mathrm{Gr}-1^{+}$IMCs. ${ }^{14,56}$ Modulation of histamine receptors through its antagonist modifies colorectal cancer progression. ${ }^{57-59}$ We observed suppression of splenic (not bone marrow) IMCs in germ-free mice colonized with WT $L$. reuteri. This suppression could be due to reduced IL-6 expression in intestinal mucosa because increased IL-6 was previously shown to increase splenic IMC infiltration. ${ }^{14,60}$ Suppression of $I L-6$ facilitates maturation of IMCs. ${ }^{14,36}$ 
However, $\triangle d g k A$ Lr colonized mice did not suppress splenic IMCs or reduce $I L-6 \mathrm{mRNA}$ expression, suggesting that $d g k A$ is important in alteration of H1R-mediated signaling and maturation of myeloid cells in peripheral compartments. Bacterial genes $h d c A$ and $d g k A$ are important for $L$. reuteri to generate sustained immunosuppressive effects in the intestine. In addition to reduced $I L-6$ gene levels we also observed reduced IL- $1 \alpha$ in blood plasma of mice that received WT $L$. reuteri compared with the GF control mice. Interestingly, $\Delta d g k A \mathrm{Lr}$ colonized mice had increased levels of plasma IL- $1 \alpha$, a proinflammatory cytokine predominantly secreted by macrophages. ${ }^{61}$ IL- $1 \alpha$ may promote IL- 6 production. ${ }^{62}$ As we observed decreased IL- $1 \alpha$ protein levels in blood plasma of WT $\mathrm{Lr}$ colonized mice but not in $\triangle h d c A$ and $\Delta d g k A$ Lr colonized mice, we speculate that luminal histamine and luminal DAG kinase may both be important for IL- $1 \alpha$ production by mucosal macrophages. Furthermore, we observed diminished mucosal IL-6 mRNA levels in the presence of WT Lr 6475 with increase in microRNA (mmu-miR)-669k-3p of BALB/c mice. Therefore, we speculate that mmu-miR-669k-3p expression modulates $I L-6$ gene expression and may have an impact on myeloid cell maturation. However, detailed studies are required to further characterize the microRNA mmu-miR-669k-3p on intestinal immunomodulation.

The mammalian microbiome includes dozens of bacterial taxa that possess the genetic capacity to perform intestinal luminal conversion of diverse biological compounds. These compounds may be derived from the diet or mammalian cells and provide substrates for bacterial enzymes. The model commensal microbe, $L$. reuteri, has yielded insights regarding luminal conversion by gut microbes and effects on epithelial and immune cell signaling pathways. Gut lactobacilli may generate small amines like histamine from amino acids and suppress inflammation via specific receptors. Gut microbes may also provide enzymes that convert lipid signals, thereby resulting in diminished signaling via receptors that promote allergic inflammation. The net effect is suppression of intestinal inflammation. Future advances in microbiome science may lead to new therapeutics that target bacterial enzymes acting in bacterial cells or mammalian cells (or both cell types) to alter cell signal or immune responses. Probiotics generating "microbial antihistamine factors" like DAG kinase may abrogate intestinal inflammation and promote the development of new microbiome-harvested treatment strategies.

\section{METHODS}

Bacterial strains. The bacterial strains used in this study were: $L$. reuteri 6475 (or also known as MM4-1A), $\Delta h d c A \operatorname{Lr} 6475$, and $\Delta d g k A$ Lr 6475. All strains were routinely cultured at $37^{\circ} \mathrm{C}$ in MRS (Man, Rogosa, and Sharpe) or LDM4 media and incubated overnight under strictly anoxic conditions using $\mathrm{N}_{2} / \mathrm{CO}_{2}(80: 20$; v/v) as the gas phase.

Gnotobiotic mouse model: bacterial colonization studies. GF $\mathrm{BALB} / \mathrm{c}$ mice (five males and five females per group) were bred in Trexler-type isolators. After weaning, all mice (10 weeks old) were colonized by gavaging with $L$. reuteri 6475 (WT strain), $\Delta$ hdcA Lr 6475 ( $h d c A$ mutant strain), or $\Delta d g k A \operatorname{Lr} 6475$ (dgkA mutant strain) $\left(1 \times 10^{9}\right.$ bacterial cells) in the presence of a $4 \% \mathrm{~L}$-histidine diet on day 1 and were killed on day 23. All mouse experiments were performed in a germ-free animal facility, according to an institutional animal care and use committee-approved mouse protocol at Baylor College of Medicine Houston, TX, and the Massachusetts Institute of Technology Committee on Animal Care (for elaborative protocols see Supplementary Text).

Immunoblot studies of pPKC signaling. Proteins were extracted from tissue samples in RIPA buffer. A total of 30 or $20 \mu \mathrm{g}$ of protein from each sample was separated by sodium dodecyl sulfatepolyacrylamide gel electrophoresis and transferred onto nitrocellulose membranes. Membranes were incubated with specific primary antibodies, nonphosphorylated $\mathrm{PKC}^{63}$ and $\mathrm{pPKC} \alpha$ (Ser 657) (diluted 1:1,000) (Santa Cruz Biotechnology, Santa Cruz, CA).

Statistics. Data were tested for normal distribution using the Kolmogorov-Smirnov test. Normally distributed data are presented as means with s.e., whereas the medians with their range are given for nonnormally distributed data. Significance of differences between Control, WT Lr, $\Delta h d c A$, and $\Delta d g k A$ mutant Lr colonized BALB/c mice were analyzed using the one-way analysis of variance test for normally distributed data (or) the Kruskal-Wallis test for nonnormally distributed data, followed by either Bonferroni/Tukey or Dunn's comparison post hoc tests. Differences between with and without DAG-treated bacterial culture gene expression and SW mice with and without WT Lr were analyzed using Student's $t$-test followed by the Mann-Whitney test for nonnormally distributed data. Differences between the groups were considered significant at ${ }^{\star} P<0.05$, ${ }^{* *} P<0.01$, and ${ }^{* * *} P<0.001$. SPSS 16.0 (IBM, Armonk, NY) for Windows 7 was used for data analysis. Prism 5.0 software (Graph Pad Software, La Jolla, CA) for Windows was used for data presentation and also for data analysis.

SUPPLEMENTARY MATERIAL is linked to the online version of the paper at http://www.nature.com/mi

\section{ACKNOWLEDGMENTS}

This work was supported by the National Institutes of Health (R01 AT004326, UH3 DK083990, and U01 CA170930 to J.V.; T32-OD010978-26, R01OD011141, and P30-ES002109 to J.G.F). Additional support was provided by NIH (National Institute for Diabetes and Digestive and Kidney Diseases)funded Texas Medical Center Digestive Diseases Center (DK56338), and unrestricted research support from BioGaia $A B$ (Stockholm, Sweden, to J.V.). We thank Eamonn Connolly and Stefan Roos (BioGaia AB, Stockholm) for providing human-derived $L$. reuteri strains, Dr Mary Estes Baylor College of Medicine (BCM) core for providing human enteroids, and Dr Hon-Chiu Leung (BCM) for assisting with protein identification by mass spectrometry. We thank James Barrish for assistance with electron microscopy, Alamelu Venkatachalam for helping with ViiA7 RT PCR machine calibration, and Karen Prince for assisting with graphics.

\section{AUTHOR CONTRIBUTIONS}

B.P.G. was involved in the study design, completion of all experiments, data analysis, and interpretation and manuscript preparation. A.H. contributed to the dgkA mutant $L$. reuteri. S.A. helped with western blot analysis and scientific discussion. J.W.N. took part in mRNA isolation and scientific discussion. R.F. took part in flow analysis. A.H. performed histamine quantification by MS. M.E. and M.L. helped with tissue harvesting. A.M. performed all the fluorescence immunohistochemistry staining. S.V. performed the MAGPIX assay. M.W. helped with Swiss Webster animal experimental set up at the Massachusetts Institute of Technology (MIT), Cambridge. M.W., J.G.F., and J.V. provided guidance, helped to design the experiments, and wrote the manuscript.

\section{DISCLOSURE}

J.V. receives unrestricted research support from BioGaia AB. The other authors declared no conflict of interest.

Official journal of the Society for Mucosal Immunology 


\section{REFERENCES}

1. Sakane, F., Imai, S., Kai, M., Yasuda, S. \& Kanoh, H. Diacylglycerol kinases: why so many of them?. Biochim. Biophys. Acta 1771, 793-806 (2007).

2. Merida, I., Avila-Flores, A. \& Merino, E. Diacylglycerol kinases: at the hub of cell signalling. Biochem. J. 409, 1-18 (2008).

3. van Blitterswijk, W.J. \& Houssa, B. Properties and functions of diacylglycerol kinases. Cell. Signal. 12, 595-605 (2000).

4. Wang, Q.J. PKD at the crossroads of DAG and PKC signaling. Trends Pharmacol. Sci. 27, 317-323 (2006).

5. Kennedy, L., Hodges, K., Meng, F., Alpini, G. \& Francis, H. Histamine and histamine receptor regulation of gastrointestinal cancers. Transl. Gastrointest. Cancer 1, 215-227 (2012).

6. Asaoka, Y., Nakamura, S., Yoshida, K. \& Nishizuka, Y. Protein kinase C, calcium and phospholipid degradation. Trends Biochem. Sci. 17, 414-417 (1992).

7. Parekh, D.B., Ziegler, W. \& Parker, P.J. Multiple pathways control protein kinase C phosphorylation. EMBO J. 19, 496-503 (2000).

8. Miller, D.J., Jerga, A., Rock, C.O. \& White, S.W. Analysis of the Staphylococcus aureus DgkB structure reveals a common catalytic mechanism for the soluble diacylglycerol kinases. Structure 16, 10361046 (2008).

9. Poli, A., Mongiorgi, S., Cocco, L. \& Follo, M.Y. Protein kinase C involvement in cell cycle modulation. Biochem. Soc. Trans. 42, 1471-1476 (2014).

10. Walter, J., Britton, R.A. \& Roos, S. Host-microbial symbiosis in the vertebrate gastrointestinal tract and the Lactobacillus reuteri paradigm. Proc. Natl. Acad. Sci. USA 108 (Suppl 1), 4645-4652 (2011).

11. Jerga, A., Lu, Y.J., Schujman, G.E., de Mendoza, D. \& Rock, C.O. Identification of a soluble diacylglycerol kinase required for lipoteichoic acid production in Bacillus subtilis. J. Biol. Chem. 282, 21738-21745 (2007).

12. Thurmond, R.L., Gelfand, E.W. \& Dunford, P.J. The role of histamine H1 and $\mathrm{H} 4$ receptors in allergic inflammation: the search for new antihistamines. Nat. Rev. Drug Discov. 7, 41-53 (2008).

13. Maintz, L. \& Novak, N. Histamine and histamine intolerance. Am. J. Clin. Nutr. 85, 1185-1196 (2007).

14. Yang, X.D. et al. Histamine deficiency promotes inflammation-associated carcinogenesis through reduced myeloid maturation and accumulation of CD11b + Ly6G + immature myeloid cells. Nat. Med. 17, 87-95 (2011).

15. Fabbri, R. et al. Memory retrieval of inhibitory avoidance requires histamine $\mathrm{H} 1$ receptor activation in the hippocampus. Proc. Natl. Acad. Sci. USA 113, E2714-E2720 (2016).

16. Hemarajata, P., Spinler, J.K., Balderas, M.A. \& Versalovic, J. Identification of a proton-chloride antiporter (EriC) by Himar1 transposon mutagenesis in Lactobacillus reuteri and its role in histamine production. Antonie Van Leeuwenhoek 105, 579-592 (2014).

17. Thomas, C.M. et al. Histamine derived from probiotic Lactobacillus reuteri suppresses TNF via modulation of PKA and ERK signaling. PLOS ONE 7, e31951 (2012).

18. Casas, I.A. \& Dobrogosz, W.J. Validation of the probiotic concept: Lactobacillus reuteri confers broad-spectrum protection against disease in humans and animals. Microb. Ecol. Health Dis. 12, 247285 (2000).

19. Spinler, J.K. et al. From prediction to function using evolutionary genomics: human-specific ecotypes of Lactobacillus reuteri have diverse probiotic functions. Genome Biol. Evol. 6, 1772-1789 (2014).

20. Liu, Y., Fatheree, N.Y., Mangalat, N. \& Rhoads, J.M. Human-derived probiotic Lactobacillus reuteri strains differentially reduce intestinal inflammation. Am. J. Physiol. Gastrointest. Liver Physiol. 299, G1087G1096 (2010).

21. Schreiber, O. et al. Lactobacillus reuteri prevents colitis by reducing P-selectin-associated leukocyte- and platelet-endothelial cell interactions. Am. J. Physiol. Gastrointest. Liver Physiol. 296, G534-G542 (2009).

22. Preidis, G.A. et al. Host response to probiotics determined by nutritional status of rotavirus-infected neonatal mice. J. Pediatr. Gastroenterol. Nutr. 55, 299-307 (2012).

23. Ferstl, R., Akdis, C.A. \& O'Mahony, L. Histamine regulation of innate and adaptive immunity. Front. Biosci. 17, 40-53 (2012).

24. Sander, L.E. et al. Selective expression of histamine receptors H1R, H2R, and $\mathrm{H} 4 \mathrm{R}$, but not $\mathrm{H} 3 \mathrm{R}$, in the human intestinal tract. Gut 55, 498-504 (2006).
25. Lieberman, P. The basics of histamine biology. Ann. Allergy Asthma Immunol. 106, S2-S5 (2011).

26. Chen, $\mathrm{X}$. et al. PKC-dependent phosphorylation of the $\mathrm{H} 1$ histamine receptor modulates TRPC6 activity. Cells 3, 247-257 (2014).

27. Mizuguchi, H. et al. Involvement of protein kinase Cdelta/extracellular signal-regulated kinase/poly(ADP-ribose) polymerase-1 (PARP-1) signaling pathway in histamine-induced up-regulation of histamine $\mathrm{H} 1$ receptor gene expression in HeLa cells. J. Biol. Chem. 286, 30542-30551 (2011).

28. Megson, A.C., Walker, E.M. \& Hill, S.J. Role of protein kinase Calpha in signaling from the histamine $\mathrm{H}(1)$ receptor to the nucleus. Mol. Pharmacol. 59, 1012-1021 (2001).

29. Urushidani, T. \& Forte, J.G. Signal transduction and activation of acid secretion in the parietal cell. J. Membrane Biol. 159, 99-111 (1997).

30. Smolinska, S. et al. Histamine receptor 2 is required to suppress innate immune responses to bacterial ligands in patients with inflammatory bowel disease. Inflamm. Bowel Dis. 22, 1575-1586 (2016).

31. Terrin, G. et al. Ranitidine is associated with infections, necrotizing enterocolitis, and fatal outcome in newborns. Pediatrics 129, e40-e45 (2012).

32. Guillet, R. et al. Association of H2-blocker therapy and higher incidence of necrotizing enterocolitis in very low birth weight infants. Pediatrics 117 , e137-e142 (2006).

33. Juillerat, P., Schneeweiss, S., Cook, E.F., Ananthakrishnan, A.N., Mogun, H. \& Korzenik, J.R. Drugs that inhibit gastric acid secretion may alter the course of inflammatory bowel disease. Aliment. Pharmacol. Ther. 36, 239-247 (2012).

34. Gao, C. et al. Histamine $\mathrm{H} 2$ receptor-mediated suppression of intestinal inflammation by probiotic Lactobacillus reuteri. mBio 6, e01358-01315 (2015).

35. Zwadlo-Klarwasser, G. et al. Generation and subcellular distribution of histamine in human blood monocytes and monocyte subsets. Inflamm. Res. 47, 434-439 (1998).

36. Ruffell, B. \& Coussens, L.M. Histamine restricts cancer: nothing to sneeze at. Nat. Med. 17, 43-44 (2011).

37. Savkovic, S.D., Koutsouris, A. \& Hecht, G. PKC zeta participates in activation of inflammatory response induced by enteropathogenic E. coli. Am. J. Physiol. Cell Physiol. 285, C512-C521 (2003).

38. Smith, R.L., O’Toole, J.F., Maguire, M.E. \& Sanders, C.R. 2nd. Membrane topology of Escherichia coli diacylglycerol kinase. J. Bacteriol. 176, 5459-5465 (1994).

39. Johansson, M.E., Larsson, J.M. \& Hansson, G.C. The two mucus layers of colon are organized by the MUC2 mucin, whereas the outer layer is a legislator of host-microbial interactions. Proc. Natl. Acad. Sci. USA 108 ((Suppl 1)), 4659-4665 (2011).

40. Ganesh, B.P., Klopfleisch, R., Loh, G. \& Blaut, M. Commensal Akkermansia muciniphila exacerbates gut inflammation in Salmonella Typhimurium-infected gnotobiotic mice. PLOS ONE 8, e74963 (2013).

41. Gao, C. et al. Microbiome-mediated suppression of inflammation associated colon carcinogenesis by histamine production in Lactobacillus reuteri. Am. J. Pathol. (2017).

42. Xie, H. \& He, S.H. Roles of histamine and its receptors in allergic and inflammatory bowel diseases. World J. Gastroenterol. 11, 2851-2857 (2005).

43. Shahid, M., Tripathi, T., Sobia, F., Moin, S., Siddiqui, M. \& Khan, R.A. Histamine, histamine receptors, and their role in immunomodulation: an updated systematic review. Open Immunol. J. 2, 9-41 (2009).

44. Natalini, P.M., Mateos, M.V., llincheta de Boschero, M.G. \& Giusto, N.M. A novel light-dependent activation of DAGK and PKC in bovine photoreceptor nuclei. Exp. Eye Res. 125, 142-155 (2014).

45. Nishizuka, Y. Protein kinase $C$ and lipid signaling for sustained cellular responses. FASEB J. 9, 484-496 (1995).

46. Mascia, F., Denning, M., Kopan, R. \& Yuspa, S.H. The black box illuminated: signals and signaling. J. Invest. Dermatol. 132, 811-819 (2012).

47. Luo, B., Regier, D.S., Prescott, S.M. \& Topham, M.K. Diacylglycerol kinases. Cell. Signal. 16, 983-989 (2004).

48. Kanoh, H., Yamada, K. \& Sakane, F. Diacylglycerol kinases: emerging downstream regulators in cell signaling systems. J. Biochem. 131, 629-633 (2002). 
49. Topham, M.K. \& Prescott, S.M. Diacylglycerol kinases: regulation and signaling roles. Thromb. Haemost. 88, 912-918 (2002).

50. Preiss, J. et al. Quantitative measurement of sn-1,2-diacylglycerols present in platelets, hepatocytes, and ras- and sis-transformed normal rat kidney cells. J. Biol. Chem. 261, 8597-8600 (1986).

51. Besterman, J.M., Pollenz, R.S., Booker, E.L. Jr. \& Cuatrecasas, P. Diacylglycerol-induced translocation of diacylglycerol kinase: use of affinity-purified enzyme in a reconstitution system. Proc. Natl. Acad. Sci. USA 83, 9378-9382 (1986).

52. Luo, B., Prescott, S.M. \& Topham, M.K. Association of diacylglycerol kinase zeta with protein kinase $C$ alpha: spatial regulation of diacylglycerol signaling. J. Cell Biol. 160, 929-937 (2003).

53. Bath, K., Roos, S., Wall, T. \& Jonsson, H. The cell surface of Lactobacillus reuteri ATCC 55730 highlighted by identification of 126 extracellular proteins from the genome sequence. FEMS Microbiol. Lett. 253, 75-82 (2005).

54. Sriramulu, D.D. et al. Lactobacillus reuteri DSM 20016 produces cobalamin-dependent diol dehydratase in metabolosomes and metabolizes 1,2-propanediol by disproportionation. J. Bacteriol. 190, 4559-4567 (2008).

55. Contreras, F.X., Sanchez-Magraner, L., Alonso, A. \& Goni, F.M. Transbilayer (flip-flop) lipid motion and lipid scrambling in membranes. FEBS Lett. 584, 1779-1786 (2010).

56. Yan, B. et al. IL-6 cooperates with G-CSF to induce protumor function of neutrophils in bone marrow by enhancing STAT3 activation. J. Immunol. 190, 5882-5893 (2013).

57. Adams, W.J. \& Morris, D.L. Short-course cimetidine and survival with colorectal cancer. Lancet 344, 1768-1769 (1994).

58. Kelly, M.D. et al. Randomized trial of preoperative cimetidine in patients with colorectal carcinoma with quantitative assessment of tumor-associated lymphocytes. Cancer 85, 1658-1663 (1999).
59. Reynolds, J.L., Akhter, J. \& Morris, D.L. In vitro effect of histamine and histamine $\mathrm{H} 1$ and $\mathrm{H} 2$ receptor antagonists on cellular proliferation of human malignant melanoma cell lines. Melanoma Res. 6, 95-99 (1996).

60. Grivennikov, S. et al. IL-6 and Stat3 are required for survival of intestinal epithelial cells and development of colitis-associated cancer. Cancer Cell 15, 103-113 (2009).

61. Shalhoub, J., Falck-Hansen, M.A., Davies, A.H. \& Monaco, C. Innate immunity and monocyte-macrophage activation in atherosclerosis. J. Inflamm. 8, 9 (2011).

62. Deon, D. et al. Cross-talk between IL-1 and IL-6 signaling pathways in rheumatoid arthritis synovial fibroblasts. J. Immunol. 167, 5395-5403 (2001).

63. Dissanayake, S.K. \& Weeraratna, A.T. Detecting PKC phosphorylation as part of the Wnt/calcium pathway in cutaneous melanoma. Methods Mol. Biol. 468, 157-172 (2008).

(i) (2) (2) This work is licensed under a Creative Commons Attribution-NonCommercial-ShareAlike $\quad 4.0$ International License. The images or other third party material in this article are included in the article's Creative Commons license, unlessindicated otherwise in thecreditline; if the material is not included under the Creative Commons license, users will need to obtain permission from the license holder to reproduce the material. To view a copy of this license, visit http:// creativecommons.org/licenses/by-nc-sa/4.0/

(C) The Author(s) 2018 